\title{
Osteotomia de nivelamento do platô tibial no tratamento da ruptura do ligamento cruzado cranial - estudo clínico em cães
}

\section{Tibial plateau leveling osteotomy on the treatment cranial cruciate ligament rupture - clinic study in dogs}

\author{
Renato Moran Ramos, ${ }^{*}$ Mônica Jorge Luz, ${ }^{*}$ Daniela Fantini Vale, ${ }^{*}$ Giseli dos Santos Ferreira, ${ }^{* *}$ Gabriela Machado, ${ }^{* * *}$ \\ Antonio Filipi Braga da Fonseca, ${ }^{* * * *}$ André Lacerda de Abreu Oliveira ${ }^{* * * *}$
}

\begin{abstract}
Resumo
A ruptura do ligamento cruzado cranial (RLCC) é a causa comum de claudicação no membro pélvico em cães. Estudos recentes demonstram que o ângulo de inclinação do platô tibial está associado à RLCC. A partir dessa descoberta, desenvolveu-se a técnica de osteotomia e nivelamento do platô tibial (TPLO). A técnica consiste na osteotomia, rotação e estabilização da porção proximal da tíbia, alterando a mecânica da articulação, neutralizando o impulso tibial cranial. A proposta do presente estudo é revisar a técnica de TPLO, enfatizando o procedimento, técnica cirúrgica, cuidados pósoperatórios e complicações. O grupo estudado era composto de 40 cães. Dezessete pacientes apresentaram RLCC no membro pélvico esquerdo e 23 no membro pélvico direito. Os ângulos do platô tibial variaram de $16^{\circ}-36^{\circ}$. Trinta animais (75\%) voltaram a apoiar o membro operado nos primeiros sete dias, enquanto seis animais (15\%) apoiaram até o $15^{\circ}$ dia, um animal $(2,5 \%)$ apoiou até o $21^{\circ}$ dia e outro $(2,5 \%)$ até o $28^{\circ}$ dia. Três animais $(7,5 \%)$ apresentaram complicações pósoperatórias, sendo elas: deiscência de sutura, fratura tibial, falha no implante. A técnica de TPLO mostrou-se eficaz no tratamento de cães com diferentes pesos com RLCC. O apoio precoce do membro afetado ocorreu na maioria dos animais. As complicações encontradas, que envolviam o procedimento cirúrgico, puderam ser corrigidas.
\end{abstract}

Palavras-chave: osteotomia, ligamento cruzado cranial, cão.

\begin{abstract}
Cranial cruciate ligament rupture (CCLR) frequently causes hindlimb limping in dogs. Recent studies showed that tibial plateau angle shift is associated with CCLR. Based on this founding the tibial plateau leveling osteotomy (TPLO) technique was developed. It consists about osteotomy, rotation and stabilization of tibial proximal portion, which modify joint mechanics thus neutralizing tibial cranial thrust. The proposal of this study is to report a review of the TPLO procedure, emphasizing procedure, surgical technique, post operative care and complications. Fourty dogs presenting CCLR were submitted to surgery, seventeen with the left limb affected, 23 on the right. The tibial plateau angles before surgery varied from $16^{\circ}$ to $36^{\circ}$. Thirty animals $(75 \%)$ returned to limb normal use in a week; six animals $(15 \%)$ were back to normal deambulation to the fifteenth day, one animal $(2,5 \%)$ were back to normal deambulation to the $20^{\circ}$ day other one before the $30^{\circ}$ day. Three animals $(7,5 \%)$ had surgical post-operatory complications, including suture descending, tibial fracture and implant failure. TPLO technique seemed appropriate for treatment of CCLR from dogs within a broad weight range. Early limb use occurred in most animals. Eventual problems arose from surgical procedure, and were promptly corrected.
\end{abstract}

Keywords: osteotomy, cranial cruciate ligament, dog.

\section{Introdução}

O ligamento cruzado cranial previne a hiperextensão do joelho, limita a rotação excessiva da tíbia em direção à face medial do fêmur, assim como evita o deslizamento cranial da tíbia em relação ao fêmur, conhecido como "movimento de gaveta", presente nos animais portadores de ruptura do ligamento cruzado cranial, reforçando sua importância na estática articular (Adams, 1986).
Esse ligamento divide-se em bandas craniomedial e caudolateral, com diferentes pontos de inserção do platô tibial. (Johnson e Hulse, 2002).

A origem da ruptura do ligamento cruzado cranial (LCC) pode ser traumática e/ou degenerativa e, ao contrário da lesão aguda, na forma crônica a ruptura do ligamento é gradativa e geralmente ocorre durante a atividade normal ou em decorrência de trauma menor (Bennett et al., 1988). Fatores

\footnotetext{
* Pós-graduação em Ciência Animal (Mestrado) Universidade Estadual do Norte Fluminense Darcy Ribeiro - UENF. Av. Alberto Lamego, 2000, Parque Califórnia, Campos dos Goytacazes.

** Pós-graduação em Ciência Animal (Doutorado) - UENF.

*** Médica-veterinária autônoma.

**** Departamento de Fisiologia e Farmacologia - UFF.

*****Departamento de Cirurgia de Pequenos animais - UENF.

A quem enviar correspondência: Renato Moran Ramos - renatomoran@hotmail.com
} 
associados à RLCC crônica incluem obesidade, envelhecimento, alterações da conformação corpórea e artropatias imunomediadas (Dupuis e Harari, 1993; Galloway e Lester, 1995; Vasseur et al., 1985). Piermattei et al., (2009), associam ainda à ocorrência de RLCC consequente a presença de uma patela instável.

Segundo Whitehair et al. (1993) e Duval et al. (1999), a RLCC ocorre mais em cães de raças grandes do que em raças pequenas.

Wingfield et al., (2000) realizaram estudo comparando as propriedades físicas do ligamento cruzado cranial do Rottweiler e do Greyhound, concluindo que em proporção com o peso corporal, a resistência do LCC dos Rottweiler é significativamente menor do que a dos Greyhoud. Piermattei et al., (2009), por sua vez, sugerem um maior acometimento pela afecção em cães de membros pouco angulados, como por exemplo o Labrador Retriever.

Recentemente foi demonstrado que o ângulo de inclinação do platô tibial está associado à ruptura do ligamento cruzado cranial (Lazar et al., 2005).

É comum em cães o comprometimento bilateral da articulação (Johnson e Hulse, 2002). Cerca de 30\% dos animais podem apresentar a RLCC contralateral em um período de dois anos (Piermattei et al., 2009).

A ruptura deste ligamento traz diversos graus de instabilidade durante toda amplitude de movimento (Arnoczky et al., 1979; Caporn e Roe 1996).

A instabilidade causada pela lesão do ligamento aumenta o deslocamento cranial da tíbia e diminui a estabilidade articular, trazendo alterações degenerativas graves nas articulações acometidas (Romano et al., 2006).

O diagnóstico da RLCC pode ser obtido através de uma avaliação da estabilidade do joelho por meio do teste de "gaveta" cranial e teste de compressão tibial ou por ambos os testes. Também se pode fazer uso do diagnóstico por imagem, tais como radiografias, artroscopia, tomografia computadorizada, ressonância magnética (Harasen, 2002).

O tratamento das lesões do LCC pode ser conservador ou cirúrgico. A seleção do tratamento leva em consideração aspectos como peso corporal, função e temperamento do paciente, tempo de evolução da lesão, custo e a preferência do cirurgião (Piermattei et al., 2009).

Métodos conservativos para o tratamento da RLCC consistem na restrição da atividade física e na utilização de analgésicos, porém, o sucesso do tratamento depende do restabelecimento da estabilidade articular (Chiericheti et al., 2001).

Os resultados desta terapia têm sido satisfatórios na maioria dos cães de pequeno porte, com peso corpóreo inferior a 15 $\mathrm{kg}$ neste caso, certo grau de estabilidade é alcançado pela fibrose periarticular, o que em pacientes relativamente pequenos é suficiente para que usem o membro afetado normalmente (Dupuis e Hahari, 1993; Vasseur, 2007; Piermattei et al., 2009). No entanto, as alterações degenerativas progridem na maioria destes animais. Cães de grande porte não respondem a esse tipo de terapia, sendo então beneficiados pela terapia cirúrgica (Piermattei et al., 2009).

O sulfato de condroitina reduz a velocidade das alterações ósseas radiográficas compatíveis com osteoartrite induzidas experimentalmente em joelho de cães, dentro de 30 a 60 dias de tratamento. A reconstrução ligamentar associada ao sulfato de condroitina promove melhora rápida na função do membro quando comparada com a reconstrução isoladamente. A manutenção do perímetro da coxa ocorreu naqueles animais que tiveram uma melhor função do membro após a correção cirúrgica (Biasi et al., 2005).

As técnicas cirúrgicas para a correção do LCC são divididas em extra-articulares e intra-articulares (Moore e Read, 1996).

Os procedimentos extra-articulares envolvem uma grande variedade de técnicas de estabilização. A maioria utiliza suturas de grosso calibre para diminuir a instabilidade articular, embora algumas confiem na transposição de tecidos moles ou ósseos (Piermattei et al., 2009), ou na utilização de tecidos biológicos (Aiken et al., 1992).

As técnicas intra-articulares visam à reconstrução do LCC rompido através da utilização de tecidos biológicos, materiais sintéticos, ou a combinação de tecidos biológicos e materiais sintéticos (Vasseur, 2007). Frequentemente as técnicas intraarticulares são realizadas em cães de grande porte ou em cães atléticos (Shires, 1993).

Recentemente surgiram novas técnicas baseadas em osteotomias corretivas para a correção da RLCC. Estas técnicas procuram alterar a mecânica da articulação para obter estabilidade pela restrição ativa da articulação do joelho. Entre as técnicas de osteotomias corretivas, destaca-se a técnica de osteotomia e nivelamento do platô tibial ("Tibial Plateau Leveling Osteotomy - TPLO") (Lazar et al., 2005).

A TPLO foi desenvolvida em 1993. Esta técnica consiste em uma osteotomia, rotação e estabilização da porção proximal da tíbia, alterando a mecânica da articulação, reduzindo o ângulo do platô tibial para próximo de $5^{\circ}$, obtendo estabilização pela restrição ativa da articulação do joelho, neutralizando o impulso tibial cranial, e assim eliminando a instabilidade articular durante a sustentação de peso em cães com lesões no ligamento cruzado cranial (Warzee et al., 2001).

Atualmente a TPLO é um dos procedimentos cirúrgicos mais utilizados para o tratamento da RLCC nos EUA e Europa, principalmente em cães de raças grandes (Lazar et al., 2005).

Hoelzler et al., (2005), indicam no pós-operatório a restrição de atividade física, até que ocorra a consolidação óssea, podendo realizar caminhadas com uso de guia nos três primeiros meses, seguido de aumento gradual e progressivo da atividade física. A utilização de uma Bandagem de RobertJones no período pós-operatório tem por objetivo minimizar a formação de edema e proteger a ferida (Vasseur 2007; Piermatei et al., 2009).

A porcentagem de complicações trans e pós-operatórias, citadas na literatura após a TPLO variam de $18 \%$ a $28 \%$, sendo elas: falhas nas placas e parafusos, infecções, fraturas por avulsão da crista da tíbia, fraturas de tíbia e fíbula, sarcomas associados aos implantes. (Slocum e Slocum, 1993; Priddy et al., 2003; Pacchiana et al., 2003; Kergosien et al., 2004; Boudrieau et al., 2005; Stauffer et al., 2006).

As principais desvantagens da TPLO são custo alto do equipamento, técnica cirúrgica complexa, necessitando de um alto grau de habilidade para executar a técnica e maior tempo operatório (Boudrieau 2009). 
O método de reconstrução escolhido varia com a preferência do cirurgião, pois estudos prospectivos demonstram índice de sucesso próximo de $90 \%$, independentemente da técnica utilizada (Piermattei et al., 2009).

Moore e Read (1996) observaram dano no corpo caudal do menisco medial em 50 a $75 \%$ dos pacientes com RLCC. Ralphs e Whitney (2002), sob visualização artroscópica, relataram $77 \%$ de acometimento do menisco lateral e $58 \%$ do menisco medial. Piermattei et al., (2009) indicam a artrotomia, para verificar a presença de possíveis traumatismos ou rupturas nos meniscos, assim como retirar os fragmentos de ligamentos rompidos. Entretanto, Chierichetti et al., (2001) fizeram um estudo utilizando 20 cães portadores de RLCC com peso de até 20 quilogramas. Dez deles foram submetidos à estabilização extra-articular com enxerto de fáscia lata e artrotomia exploratória, e dez foram submetidos à mesma técnica, porém sem artrotomia exploratória. A técnica realizada sem artrotomia exploratória mostrou-se mais eficiente, clínica e estatisticamente.

Neste trabalho objetiva-se discutir a técnica de TPLO e os resultados obtidos por ela, em 40 casos de RLCC, envolvendo cães de diferentes tamanhos.

\section{Material e método}

Este trabalho foi desenvolvido pelo setor de ortopedia e traumatologia do Hospital Veterinário da Universidade Estadual do Norte Fluminense - UENF.

Foram estudados 40 cães com diagnóstico de ruptura do ligamento cruzado cranial, com pesos variando entre 17-62 $\mathrm{kg}$, submetidos ao procedimento de osteotomia e nivelamento do platô tibial.

Os animais foram inicialmente avaliados quanto ao seu estado geral de saúde através de exame clínico e laboratorial, a fim de detectar alterações orgânicas importantes capazes de prejudicar os resultados do estudo, bem como comprometer a vida do paciente.

Exames radiográficos do joelho foram realizados para a aferição do ângulo do platô tibial, na projeção médio-lateral com angulação entre o fêmur e a tíbia de $90^{\circ}$ e tíbia e metatarsos em $90^{\circ}$. Os côndilos femorais foram mantidos paralelos. A técnica operatória era planejada a partir das radiografias. Os ângulos aferidos variaram de $16^{\circ}$ a $36^{\circ}$.

Para realização dos procedimentos cirúrgicos, os cães foram mantidos em jejum hídrico e alimentar nas 12 horas que antecederam o ato cirúrgico.

A medicação pré-anestésica foi realizada com acepromazina $\left(0,05 \mathrm{mg} \cdot \mathrm{kg}^{-1}\right)$ e morfina $\left(0,3 \mathrm{mg} \cdot \mathrm{kg}^{-1}\right)$ por via intramuscular a indução anestésica com propofol $\left(5 \mathrm{mg} \cdot \mathrm{kg}^{-1}\right)$, por via intravenosa. Realizou-se anestesia epidural com morfina $(0,1$ mg.kg-1), lidocaína $2 \%$ sem vasoconstritor $\left(0,2 \mathrm{ml}^{-\mathrm{kg}^{-1}}\right)$ e cloridrato de bupivacaina $0,5 \%\left(0,2 \mathrm{ml} \cdot \mathrm{kg}^{-1}\right)$. A manutenção da anestesia foi feita com anestésico volátil (isoflurano) diluído em oxigênio $100 \%$, administrado por via pulmonar pela sonda endotraqueal, em circuito semifechado.

Foi realizada a tricotomia do membro acometido. Em seguida, os animais foram posicionados em decúbito dorsal e a antissepsia foi realizada desde o terço proximal do fêmur estendendo-se até o terço distal da tíbia, com solução de polivinil pirrolidona, procedendo-se, então, o isolamento da extremidade do membro com malha tubular estéril. Foram colocados panos de campo a fim de delimitar o campo operatório na região do joelho.

Foi feito o acesso craniomedial da soldra, através de uma incisão cutânea longitudinal, $2 \mathrm{~cm}$ acima da patela, estendendo-se até o final do terço proximal da tíbia (Figura 1). Após divulsão do tecido celular subcutâneo, as inserções dos músculos grácil e semitendinoso e a bainha caudal do músculo sartório foram identificadas e seccionadas. Uma agulha $25 \times 0.7 \mathrm{~mm}$ foi inserida no espaço articular femorotibial, para identificar o espaço articular entre o fêmur e a tíbia (Figura 2). Posicionou-se a serra semicircular oscilatória transversalmente na região proximal da tíbia, com uma angulação de $90^{\circ} \mathrm{em}$ relação à face caudal e face medial da tíbia. A lâmina localizou-se caudalmente à crista tibial (Figura 3). A serra foi acionada marcando o local da osteotomia. Com um osteotomo, promoveu-se a marcação dos pontos a serem alinhados após a osteotomia. A primeira marcação foi realizada no segmento proximal da osteotomia, posicionada cranialmente à segunda marcação, que foi realizada no segmento distal (Figura 4). A jig (instrumento utilizado para manter posicionados os segmentos tibiais) foi posicionada no segmento proximal da osteotomia e na porção distal da tíbia (Figura 5). A osteotomia da tíbia foi concluída. Um pino de Steinmann foi inserido obliquamente no segmento proximal, na sua face medial, próximo à porção caudal, para que este auxiliasse no deslizamento dos dois segmentos da tíbia, alinhando-se as marcações previamente realizadas (Figura 6). Outro pino de Steinmann foi inserido através da região cranial da tíbia, ultrapassando a osteotomia, fixando os dois segmentos. A placa específica para TPLO, com três parafusos proximais e três parafusos distais, foi moldada à superfície medial da tibial, fixando os dois segmentos, mantendo desta maneira, as duas marcações alinhadas (Figura 7). Os pinos de Steinmann e a jig foram retirados. A fáscia muscular profunda foi suturada sobre a placa com o fio de náilon monofilamentado 3-0, em padrão contínuo simples. O tecido subcutâneo foi suturado com fio de náilon monofilamentado 3-0 em padrão Cushing e a pele com o mesmo fio em padrão interrompido simples. A medicação pós-operatória prescrita foi meloxicam $\left(0,1 \mathrm{mg} \cdot \mathrm{kg}^{-1}\right.$ VO SID) durante cinco dias, cefalexina (30 mg. $\mathrm{kg}^{-1} \mathrm{VO}$ BID) durante 10 dias e tramadol (2 mg. $\mathrm{kg}^{-1}$ VO TID) durante três dias.

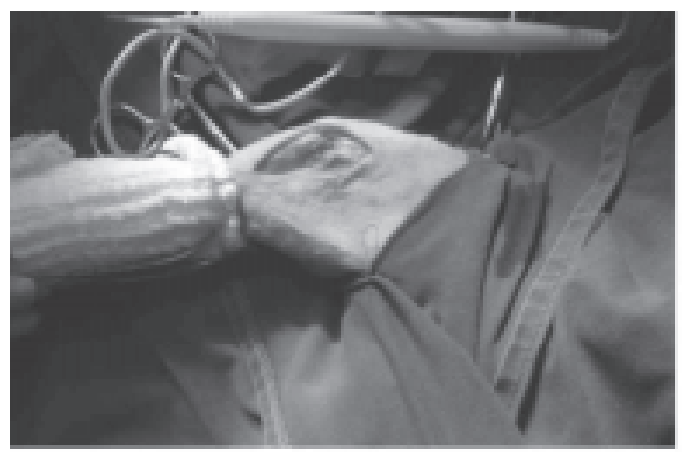

Figura 1: Incisão cutânea longitudinal, $2 \mathrm{~cm}$ acima da patela, estendendose até o final do terço proximal da tíbia. (Arquivo pessoal, Hospital Veterinário - UENF 2008) 


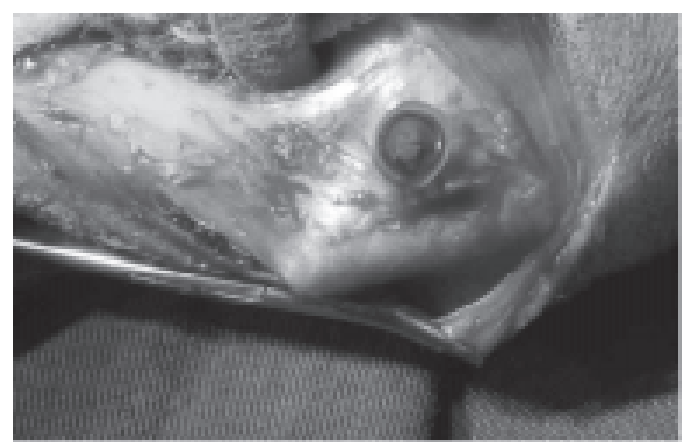

Figura 2: Agulha $25 \times 0.7 \mathrm{~mm}$ inserida no espaço articular femorotibial, para identificar o platô tibial. (Arquivo pessoal. Hospital Veterinário UENF, 2008)

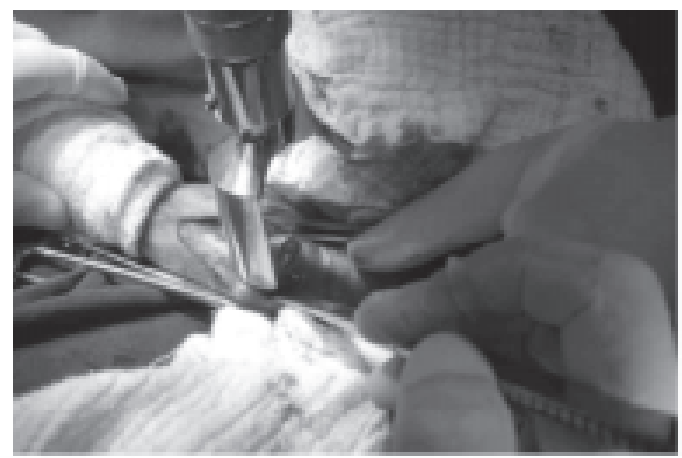

Figura 3: Serra semicircular oscilatória posicionada transversalmente na região proximal da tíbia, com uma angulação de $90^{\circ} \mathrm{em}$ relação à face caudal e face medial da tíbia. A lâmina localizou-se caudalmente à crista tibial. (Arquivo pessoal. Hospital Veterinário - UENF, 2008)

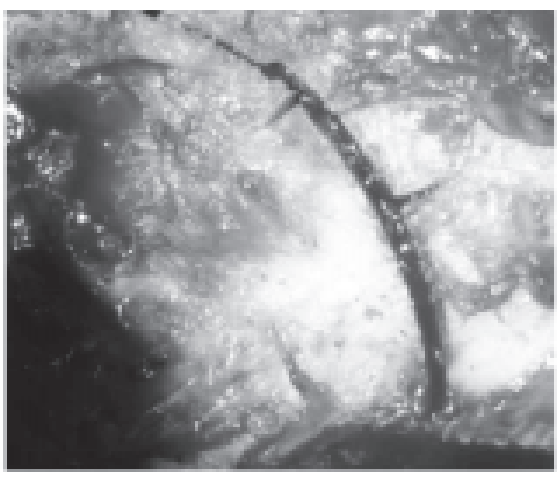

Figura 4: Primeira marcação realizada no segmento proximal da osteotomia, posicionada cranialmente à segunda marcação, que foi realizada no segmento distal. (Arquivo pessoal. Hospital Veterinário UENF, 2008)

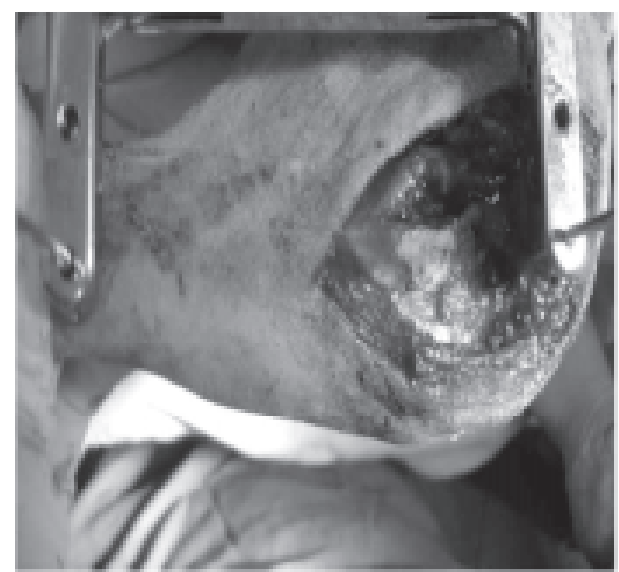

Figura 5: Jig posicionada no segmento proximal da osteotomia e na porção distal da tíbia. (Arquivo pessoal. Hospital Veterinário - UENF, 2008)

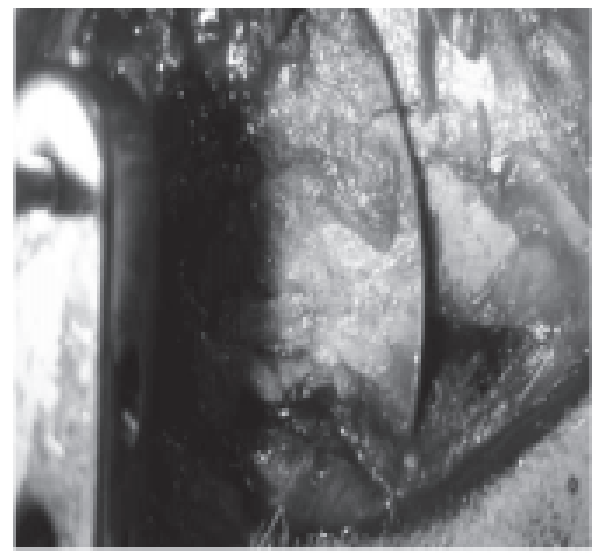

Figura 6: Pino de Steinmann foi inserido obliquamente no segmento proximal, na sua face medial, próximo à porção caudal, para que este auxilie no deslizamento dos dois segmentos da tíbia, alinhando-se às marcações previamente realizadas. Arquivo pessoal. Hospital Veterinário - UENF, 2008

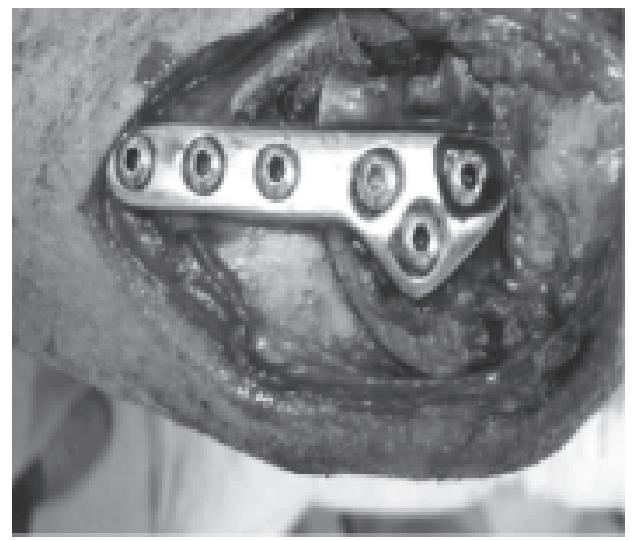

Figura 7: Placa específica para TPLO, com três parafusos proximais e três parafusos distais, moldada à superfície medial da tibial, fixando os dois segmentos, mantendo as duas marcações alinhadas. Arquivo pessoal. Hospital Veterinário - UENF, 2008 
No pós-operatório, colocou-se uma bandagem Robert Jones no membro operado. Esta foi retirada no terceiro dia de pósoperatório. Após esse período, o curativo cirúrgico foi feito com iodopovidona e coberto com micropore, sendo trocados uma vez ao dia, pelo proprietário, até a retirada dos pontos, após 10 dias. Instruiu-se o proprietário para que os cães fossem mantidos em repouso nos primeiros dois meses, permitindo-se somente caminhadas. Em seguida, indicou-se aumento gradual de atividade física, sem realização de exercícios forçados.

Foi considerado retorno do apoio do membro operado, o primeiro dia que o animal voltou a locomover-se, com apoio contínuo do membro.

\section{Resultados}

Dezessete pacientes apresentaram RLCC no membro pélvico esquerdo e 23 no membro pélvico direito. As raças acometidas foram (Bulldog inglês - 3 pacientes; Cocker Spaniel inglês - 1 paciente; Dogue Alemão - 2 pacientes; Fila Brasileiro - 4 pacientes; Golden Retriever - 2 pacientes; Labrador 11 pacientes; Pitbul - 4 pacientes; Rottweiler - 11 pacientes; Sem raça definida - 2 pacientes). Os ângulos do platô tibial variaram de $16^{\circ}-36^{\circ}$ (Quadro 1).

O intervalo entre a suspeita da lesão por parte dos proprietários e os procedimentos cirúrgicos variou entre três e cinco semanas.

No momento do diagnóstico da RLCC, os cães apresentavam claudicação com apoio intermitente do membro ou não o apoiavam. O tempo cirúrgico variou de 57 a 90 minutos, sendo a média em minutos de 74,38 \pm 1.431.

Trinta animais voltaram a apoiar o membro operado na primeira semana, enquanto seis animais apoiaram até a segunda semana, um animal apoiou até a terceira semana e outro até a quarta semana (Gráfico - 1). Três animais apresentaram complicações pós-operatórias, sendo elas: deiscência de sutura, fratura do fragmento proximal da tíbia e falha no implante. Dentre os animais que apresentaram complicações, o animal que apresentou fratura tibial foi novamente operado. Ao final desse estudo, este animal apresentava apoio intermitente do membro. O cão que apresentou falha do implante foi submetido a um novo procedimento cirúrgico, para substituição do mesmo, voltando a apoiar o membro após a $4^{\mathrm{a}}$ semana.

\begin{tabular}{|c|c|c|c|c|}
\hline Raça & $\begin{array}{c}\text { Peso } \\
(\mathrm{kg})\end{array}$ & $\begin{array}{l}\text { Membro } \\
\text { afetado }\end{array}$ & TPA & $\begin{array}{l}\text { Tempo de retorno a } \\
\text { função / complicações }\end{array}$ \\
\hline Bulldog inglês & 22,3 & $\mathrm{D}$ & $36^{\circ}$ & 1 \\
\hline Bulldog inglês & 28,7 & $E$ & $24^{\circ}$ & 1 \\
\hline Bulldog inglês & 23 & D & $26^{\circ}$ & 1 \\
\hline Cocker spaniel inglês & 17 & $\mathrm{D}$ & $20^{\circ}$ & 1 \\
\hline Dogue alemão & 58,2 & D & $20^{\circ}$ & 1 \\
\hline Dogue alemão & 60 & $E$ & $16^{0}$ & 1 \\
\hline Fila brasileiro & 62 & $\mathrm{E}$ & $20^{\circ}$ & 1 \\
\hline Fila brasileiro & 56,9 & $E$ & $24^{\circ}$ & 1 \\
\hline Fila brasileiro & 61,6 & $\mathrm{D}$ & $20^{\circ}$ & 1 \\
\hline Fila brasileiro & 52,8 & $\mathrm{E}$ & $25^{\circ}$ & Falha do implante \\
\hline Golden retriever & 25 & $\mathrm{D}$ & $20^{\circ}$ & 2 \\
\hline Golden retriever & 24,1 & $E$ & $22^{\circ}$ & 1 \\
\hline Labrador & 24,5 & $\mathrm{D}$ & $20^{\circ}$ & 1 \\
\hline Labrador & 25,5 & D & $30^{\circ}$ & 1 \\
\hline Labrador & 20 & $\mathrm{D}$ & $20^{\circ}$ & 2 \\
\hline Labrador & 22 & $\mathrm{D}$ & $24^{\circ}$ & 1 \\
\hline Labrador & 19,8 & $E$ & $16^{\circ}$ & 1 \\
\hline Labrador & 30 & $\mathrm{D}$ & $16^{\circ}$ & 1 \\
\hline Labrador & 28,3 & $\mathrm{D}$ & $23^{\circ}$ & 1 \\
\hline Labrador & 26 & $\mathrm{D}$ & $21^{\circ}$ & 1 \\
\hline Labrador & 25,5 & $\mathrm{D}$ & $23^{\circ}$ & 1 \\
\hline Labrador & 24,3 & $E$ & $21^{\circ}$ & 1 \\
\hline Labrador & 19,5 & $\mathrm{D}$ & $18^{\circ}$ & Fratura tibial \\
\hline Pit bull & 28,3 & $\mathrm{E}$ & $16^{\circ}$ & 1 \\
\hline Pit bull & 32 & $\mathrm{D}$ & $28^{\circ}$ & 1 \\
\hline Pit bull & 29 & $\mathrm{E}$ & $26^{\circ}$ & 4 \\
\hline Pit bull & 31,4 & $\mathrm{E}$ & $30^{\circ}$ & 3 \\
\hline Rottweiler & 36 & $E$ & $26^{\circ}$ & 1 \\
\hline Rottweiler & 42,2 & $\mathrm{D}$ & $18^{\circ}$ & 1 \\
\hline Rottweiler & 38 & $\mathrm{D}$ & $18^{\circ}$ & 2 \\
\hline Rottweiler & 32,4 & $E$ & $27^{\circ}$ & 1 \\
\hline Rottweiler & 31,4 & $\mathrm{D}$ & $23^{\circ}$ & 1 \\
\hline Rottweiler & 29 & $\mathrm{D}$ & $16^{\circ}$ & 1 \\
\hline Rottweiler & 27,6 & $\mathrm{E}$ & $20^{\circ}$ & 1 / Deiscência de sutura \\
\hline Rottweiler & 50,8 & $\mathrm{D}$ & $30^{\circ}$ & 1 \\
\hline Rottweiler & 42,1 & $\mathrm{E}$ & $16^{\circ}$ & 2 \\
\hline Rottweiler & 36,3 & $\mathrm{D}$ & $20^{\circ}$ & 2 \\
\hline Rottweiler & 28,7 & $E$ & $30^{\circ}$ & 1 \\
\hline Sem raça definida & 17,2 & $E$ & $20^{\circ}$ & 1 \\
\hline Sem raça definida & 18,1 & $\mathrm{D}$ & $21^{\circ}$ & 2 \\
\hline
\end{tabular}

D: Direito; E: Esquerdo; TPA: Ângulo de inclinação do platô tibial; Tempo de retorno a função do membro em semanas; Peso em quilogramas.

Quadro 1: Relação dos cães portadores de RLCC operados com a técnica TPLO, quanto à raça, ao membro acometido, ângulo de inclinação do platô tibial e tempo de retorno à função do membro / complicações 


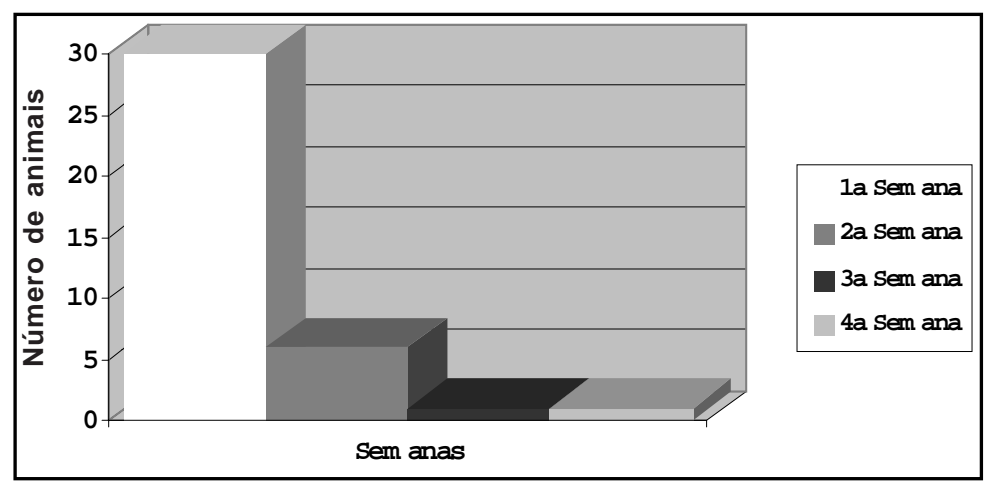

Gráfico 1: Tempo de retorno à função do membro (semanas)

\section{Discussão}

Este estudo apresenta informações importantes a respeito do uso da TPLO no Brasil, que a despeito de ser mundialmente utilizado (Guimarães, 2006), ainda não é o método mais aplicado em nosso país. Acredita-se que isso se deva ao treinamento mais laborioso para o seu aprendizado, bem como as dificuldades inerentes ao seu custo e uso de equipamentos específicos ainda não comercializados no Brasil.

A técnica de TPLO foi empregada em animais que apresentaram pesos variando entre 17 a $62 \mathrm{~kg}$, apresentando excelentes resultados, mesmo em cães de tamanhos distintos, demonstrando a versatilidade da técnica.

No presente estudo $85 \%$ dos animais são classificados como raças de porte grande ou gigante. Segundo Whitehair et al. (1993) e Duval et al. (1999), a RLCC ocorre mais em raças grandes do que em raças pequenas.

As raças mais acometidas durante este estudo foram: Rottweiler (27,5\%), seguidas do Labrador (27,5\%), Fila Brasileiro (10\%) e do Starffordshire Terrier (10\%), como relatado por Whitehair et al. (1993).

Wingfield et al. (2000) realizaram estudo comparando as propriedades físicas do ligamento cruzado cranial do Rottweiler e do Greyhound, concluindo que em proporção com o peso corporal, a resistência do LCC dos Rottweiler é significativamente menor do que a dos Greyhoud, sugerindo a predisposição dos Rottweiler para esta doença, o que já se verificava epidemiologicamente.

Piermattei et al. (2009), por sua vez, sugerem um maior acometimento pela afecção em cães de membros pouco angulados, como por exemplo o Labrador Retriever. Esta raça, juntamente com a Rottweiler, foram as que apresentaram maior prevalência da RLCC no presente trabalho.

Não se utilizou a artrotomia exploratória para a verificação de lesões meniscais, apesar de Shires (1993) e Piermattei et al. (2009) relatarem que, independentemente da técnica cirúrgica a ser escolhida, a articulação do joelho deve ser aberta, explorada e irrigada com solução salina estéril. A membrana sinovial deve ser inspecionada, osteófitos devem ser removidos e os meniscos inspecionados cuidadosamente. Este fato não levou à ocorrência de piores resultados, coincidindo com os estudos de Chierichetti et al. (2001), que obtiveram melhores resultados nos animais nos quais não foram realizadas as artrotomias.
No presente estudo, o tempo cirúrgico variou entre 57 e 90 minutos, com média e desvio-padrão de 74,38 \pm 1.431 , sendo os tempos maiores alcançados nas operações iniciais e os tempos menores nas últimas operações. Isso demonstra a ocorrência da curva de aprendizado, concordando com Boudrieau (2009), que cita como desvantagem da TPLO um alto grau de habilidade para executar a técnica.

O retorno da função do membro ocorreu precocemente, contribuindo para diminuição da perda de massa muscular no membro operado, como citado por Piermattei et al. (2009), que verificaram que o desuso do membro poderia causar atrofia muscular. Biasi et al. (2002) observaram a manutenção do perímetro da coxa naqueles animais que tiveram uma melhor função do membro após a correção cirúrgica. $\mathrm{O}$ apoio contínuo do membro operado ocorreu na primeira semana em 30 animais (75\%). Seis animais (15\%) voltaram a apoiar o membro acometido na segunda semana, um animal $(2,5 \%)$ até a terceira semana e um animal $(2,5 \%)$ na quarta semana. Baraúna Júnior et al. (2007) obtiveram o retorno funcional do membro, em média 12 semanas após o procedimento operatório, com a técnica de interligação extracapsular femorofabelo-tibial, demonstrando que a TPLO atualmente é uma técnica que apresenta excelentes resultados clínicos, com um tempo curto de retorno à função do membro.

A restrição da atividade foi requerida a todos os pacientes, como descrito por Hoelzler et al. (2005), que indicam no pósoperatório a restrição de atividade física, até que ocorra a consolidação óssea, podendo realizar caminhadas com uso de guia nos três primeiros meses, seguido de aumento gradual e progressivo da atividade física.

A utilização de uma Bandagem de Robert-Jones no período pós-operatório evitou a formação de edema, o que vem a corroborar com as indicações de Vasseur (2007) e Piermatei et al. (2009), onde citam que a utilização deste tipo de bandagem tem por objetivo minimizar o edema e proteger a ferida nas primeiras 48 horas.

Complicações foram observadas em $7,5 \%$ dos animais. Os que apresentaram fratura tibial e falha no implante foram novamente operados e voltaram a apoiar o membro após a 4ª semana. Estas complicações mais graves ocorreram em cães de raças grandes, como observaram Slocum e Slocum, (1993); Priddy et al. (2003); Pacchiana et al. (2003); Stauffer et al. (2006); Kergosien et al. (2004) e Boudrieau et al. (2005). Isso pode ter ocorrido, pois cães de grande porte podem sobrecarregar o implante, caso o repouso pós-operatório não seja rigorosamente seguido.

Em relação à técnica cirúrgica, não foram observadas complicações transoperatórias.

\section{Conclusão}

A técnica de osteotomia e nivelamento do platô tibial mostrouse eficiente e versátil, apresentando excelentes resultados, pois o apoio precoce do membro operado ocorreu em $75 \%$ dos animais, tanto para correção da ruptura do ligamento cruzado cranial em raças de pequeno e grande porte e em cães com excessivo ângulo do platô tibial, assim como apresentando um baixo índice de complicações. 


\section{Referências}

ADAMS, D.R. Canine anatomy. Ames: The lowa State University Press, 1986.

AIKEN, S.W.; BAUER, M.S.; TOOMBS, J.P. Extraarticular fascial strip repair of the cranial cruciate deficient stiffle: technique and results in seven dogs. Veterinary and Comparative Orthopaedics and Traumatology, v. 5, p.145-150, 1992.

ARNOCZKY, S.P.; RUBIN, R.N.; MARSHALL, J.L. Microvasculature of the cruciate ligaments and its response to injury. Journal of Bone e Joint Surgery. v. 61 p.1227-1229, 1979.

BIASI, F.; RAHAL, S.C.; VOLPI, R.S.; et al., Reconstrução do ligamento cruzado cranial em cães, associado ou não ao sulfato de condroitina. Arquivo Brasileiro de Medicina Veterinária e Zootecnia. v. 57, n. 4, p. 442-447, 2005.

BOUDRIEAU, R.J.; MCCARTHY, R.J.; SISSON, R.D. Jr. Sarcoma of the proximal portion of the tibia in a dog 5.5 years after tibial plateau leveling osteotomy. Journal of American Veterinary Medical Association, v. 227 p. 1613-1617, 2005.

CAPORN, T.M.; ROE, S.C. Biomechanical evaluation of the suitability of monofilament nylon fishing and leader line for extra-articular stabilization of thr canine cruciate-deficiente stifle. Veterinary and Comparative Orthopaedics and Traumatology. v. 9, p. 126-133, 1996. CHIERICHETTI, A.L.; ALVARENGA, J.; PEDRO, C.R. et al., Ruptura de ligamento cruzado cranial. Estudo comparativo da técnica extraarticular com enxerto autógeno de fáscia lata com e sem artrotomia exploratória. Clínica Veterinária. n. 33, p.34-42, 2001.

DUPUIS, J.; HARARI, J. Cruciate ligament and meniscal injuries in dogs. Compendium of Continuing Education for the Practicing Veterinarian. v. 15, n. 2, p. 215-232, 1993.

DUVAL, J. M.; BUDSBERG, S.C.; FLO, G.L.; et al., Breed, sex, and body weight as risk factors for rupture of the cranial cruciate ligament in young dogs. Journal of American Veterinary Medical Association. v. 215, n. 6, p. 811-814, 1999.

GALLOWAY, R.H.; LESTER, S.J. Histopathological evaluation of canine stifle joint synovial membrane collected at the time of repair of cranial cruciate ligament rupture. Journal of the American Animal Hospital Association. v. 31, n. 4, p. 289-294, 1995.

HARASEN, G. Diagnosing rupture of the cranial cruciate ligament Can. Veterinary Journal, v. 43, n. 6, p. 475-476, 2002.

HOELZLER, M. G.; HARVEY, R. C.; LIDBETTER, D. A.; et al., Comparison of perioperative analgesic protocols for dogs undergoing tibial plateau leveling osteotomy, Veterinary Surgery, v. 5, n. 34, p. 337-344, 2005. JOHNSON, A.L.; HULSE, D.A. Disease of the Joints In: FOSSUM, T.W. Small Animal Surgery, 2. ed., St. Louis: Mosby, p. 883-993. 2002.

KERGOSIEN, D. H.; BARNHART, M. D.; KEES, C. E.; et al., Radiographic and clinical changes of the tibial tuberosity after tibial plateau leveling osteotomy. Veterinary Surgery, Philadelphia, v. 33, n. 5, p. 468-474, 2004.

LAZAR T.P., BERRY C.R., DEHAAN J.J., et al., Long-Term Radiographic Comparison of Tibial Plateau Leveling Osteotomy Versus Extracapsular Stabilization for Cranial Cruciate Ligament Rupture in the Dog. Veterinary Surgery. v. 34. p. 133-141, 2005.
MOORE, K.W.; READ, R.A. Rupture of the cranial cruciate ligament in dogs. II, Compendium on Continuing Education for the Practicing Veterinarian. n. 18 p. 38, 1996.

PACCHIANA, P.D.; MORRIS, E.; GILLINGS, S.L.; et al., Surgical and postoperative complications associated with tibial plateau leveling osteotomy in dogs with cranial cruciate ligament rupture: 397 cases (1998-2001). Journal of American Veterinary Medical Association, v. 222, p. 184-193, 2003.

PIERMATTEI, D.L.; FLO, G.L; DECAMP, C.E. The stifle joint. In: Brinker, Piermattei, Flo Ortopedia e tratamento de Fraturas de Pequenos animais. 4. ed. Manole p. 661-688, 2009.

PRIDDY, N.H.; TOMLINSON, J.L; DODAM, J.R; et al., Complications with and owner assessment of the outcome of tibial plateau leveling osteotomy for treatment of cranial cruciate ligament rupture in dogs: 193 cases (1997-2001). Journal of American Veterinary Medical Association, v. 222, p. 1726-1732, 2003.

RALPHS, S. C.; WHITNEY, W. O. Arthroscopic evaluation of menisci in dogs with cranial cruciate ligament injuries: 100 cases (1999-2000). Journal of the American Veterinary Medical Association, v. 221, n. 11, p. 1601-1604, 2002.

ROMANO, L.; PEREIRA, C.A.M.; SCHMAEDECKE, A.; et al., Análise biomecânica do joelho íntegro e com rupturado ligamento cruzado cranial quanto ao grau de deslocamento cranial e rigidez articular em cães. Acta Cirúrgica Brasileira. Jan-Fev; v. 21, n. 1, 2006.

SHIRES, P.K. Intracapsular repairs for cranial cruciate ligament ruptures. Veterinary Clinics of North America: Small Animal Practice, v. 23, p. 761-776, 1993.

SLOCUM, B.; SLOCUM, T. D. Tibial plateau leveling osteotomy for repair of cranial cruciate ligament rupture in the canine. Veterinary Clinics of North America: Small Animal Practice, Philadelphia, v. 23, n. 4, p. 777-795, 1993.

STAUFFER, K.D.; TUTTLE, T.A.; ELKINS, A.D.; et al., Complications associated with 696 tibial plateau leveling osteotomies (2001-2003). Journal of the American Animal Hospital Association v. 42 p. 44-50, 2006.

VASSEUR, P. B.; POOL, R. R.; ARNOCZKY, S. P, et al., Correlative biomechanical and histologic study of the cranial cruciate ligament in dogs. American Journal of Veterinary Research, v. 46, n. 9, p. 18421854, 1985.

VASSEUR, P.B. Articulação do joelho. In: SLATTER, D. Manual de cirurgia de pequenos animais. São Paulo: Manole, p. 2090-2116, 2007.

WARZEE C.C.; DEJARDIN L.M.; ARNOCZKY S.P.; et al., Effect of tibial plateau leveling on cranial and caudal tibial thrusts in canine cranial cruciate-deficient stifles: an in vitro experimental study. Veterinary Surgery, v. 30 p. 278-286, 2001.

WHITEHAIR, J. G.; VASSEUR, P. B.; WILLITS, N. H. Epidemiology of cranial cruciate ligament rupture in dogs. Journal of American Veterinary Medical Association. v. 203, n. 7, p. 1016-1019,1993.

WINGFIELD C.; AMIS, A.A.; STEAD, A.C.; et al., Comparision of the biomechanical properties of Rottweiler and racing greyhound cranial cruciate ligaments. Journal of Small Animal Practice, v. 41, p. 303307,2000 . 